Supporting information

\title{
Liquid phase catalytic exchange of hydrogen isotopes over platinum on dual-modified graphene
}

\author{
Yongsheng Xu ${ }^{a}$, Feng Xin ${ }^{a}$ *, Yue Jiang ${ }^{b}$, Xiaohong Yin ${ }^{b, *}$
}

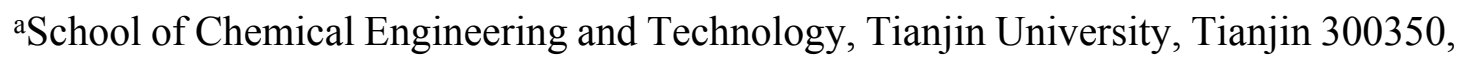
China.

bSchool of Chemistry and Chemical Engineering, Tianjin University of Technology, Tianjin, 300384, PR China.

Corresponding authors:

Feng Xin

E-mail: xinf@tju.edu.cn

Xiaohong Yin

E-mail: yinxiaohong@tjut.edu.cn 


\section{Methods}

\subsection{Preparation of GO}

The GO was synthesized by modified Hummer's method as follow steps. Firstly, $230 \mathrm{~mL}$ concentrated sulfuric acid was stirred in $2 \mathrm{~L}$ three-neck flask at $0{ }^{\circ} \mathrm{C}$. Secondly, $5 \mathrm{~g} \mathrm{NaNO}_{3}$ and $5 \mathrm{~g}$ graphite are gradually added into above system, respectively. Then, the mixture was stirred constantly and reacted with $30 \mathrm{~min}$. Thirdly, $30 \mathrm{~g} \mathrm{KMnO}_{4}$ was added into the reaction system gradually (more than 10 times). After added, the temperature of the system was increased at $35^{\circ} \mathrm{C}$ and went on the reaction with $30 \mathrm{~min}$. Fifthly, $500 \mathrm{~mL}$ deionized water was added into the reaction system gradually. Then, the temperature of system was increased to $98^{\circ} \mathrm{C}$ and went on the reaction with $60 \mathrm{~min}$. Sixthly, $1200 \mathrm{~mL}$ and $500 \mathrm{~mL} 5 \mathrm{wt} \% \mathrm{H}_{2} \mathrm{O}_{2}$ were added into the system and the solution was filtered at a high temperature. Then, the obtained solid was washed by $5 \% \mathrm{HCl}$ solution and water, respectively. Finally, the as-prepared sample was dispersed in amount of deionized water and then the suspension stirred with 1 day at room temperature. After that, the suspension was put into dialysis bags and dialysed for five days. The obtained suspension in dialysis bags was GO suspension.

\subsection{Preparation of catalyst coating}

The "PDMS+catalyst" coating was prepared on the reactor wall according to Cao's method. The procedure was specified as follows: firstly, the diluted PDMS solution ( $10 \% \mathrm{w} / \mathrm{v}$ in hexane) was prepared by dissolving PDMS base and curing agent (a 10:1 w/w ratio) in hexane. Secondly, a mount of diluted PDMS solution were

dragged into the reactor and then rolling the reactor to obtain a thin liquid film deposited on the reactor wall. After the most of hexane was evaporated, the uncured adhesive surface was covered by the catalyst powder. Finally, the prepared coating was cured under $80^{\circ} \mathrm{C}$ overnight. 


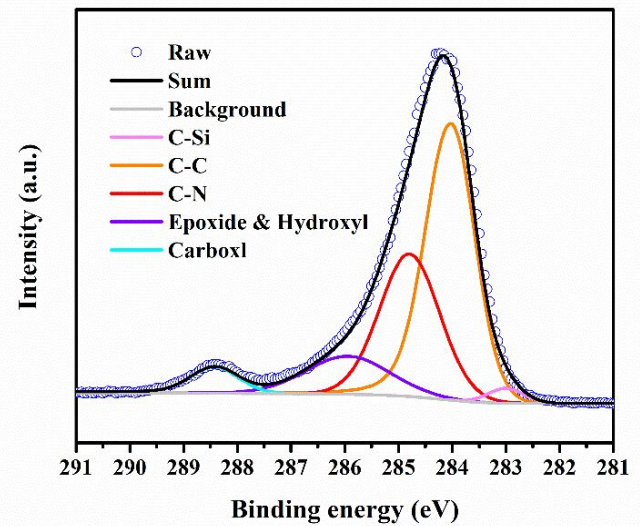

Figure S1. High-resolution spectrum of C 1s for 200-S-NH $2-G R$. 


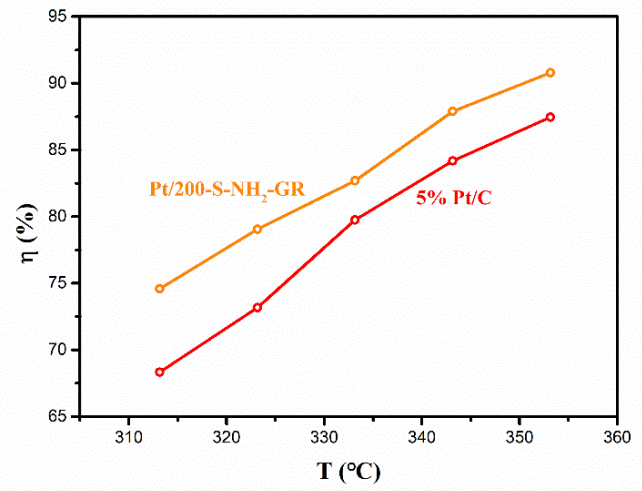

Figure S2. Catalytic performances for $\mathrm{Pt} / 200-\mathrm{S}-\mathrm{NH}_{2}-\mathrm{GR}$ and $5 \% \mathrm{Pt} / \mathrm{C}$. 


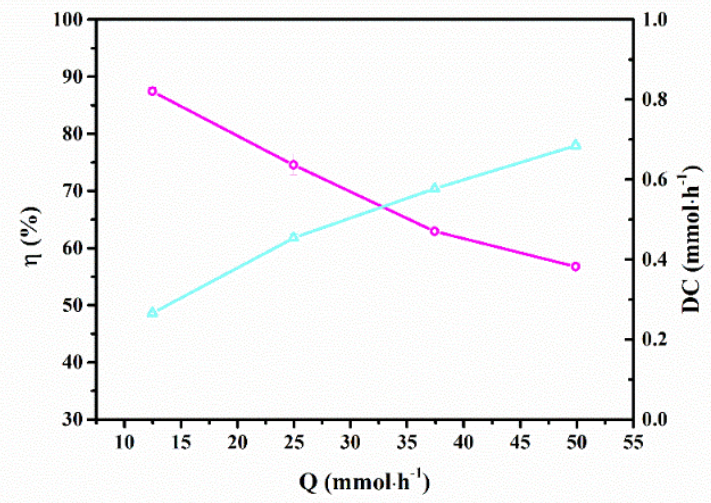

Figure S3. Catalytic performances for $\mathrm{Pt} / 200-\mathrm{S}-\mathrm{NH}_{2}-\mathrm{GR}$ under different flow rates at $40{ }^{\circ} \mathrm{C}$. 


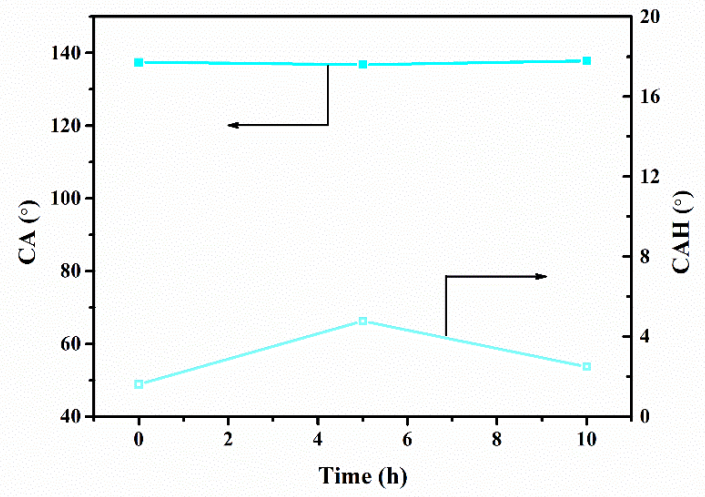

Figure S4. Stability experiment for "PDMS $+\mathrm{Pt} / \mathrm{NH}_{2}-\mathrm{GR}$ " coating $40{ }^{\circ} \mathrm{C}$. 